\title{
Simple formulas for theoretical minimum emittance in storage rings
}

\author{
Chun-xi Wang* \\ Argonne National Laboratory, 9700 South Cass Avenue, Argonne, Illinois 60439, USA
}

Wen Wei Ho

Princeton University, Princeton, New Jersey 08544, USA

(Received 28 September 2011; published 12 January 2012)

\begin{abstract}
We present an analytical calculation of the theoretical minimum emittance in storage rings with arbitrary but nonreversing bending magnets. Our derivation is based on a dipole with a short segment of constant bending radius and linear ramps at the ends, which has been shown to be very close to the optimal bending profile. The analytical results confirm and extend the previous ones obtained by numerical optimizations (except for a minor uncertainty on the profile for minimum effective emittance). Simple approximate formulas are given for calculating the theoretical minimum emittance and the parameters of the required bending profile and optics functions, which are of practical value for storage-ring designs. To facilitate designs of linear optics using the optimal bending profiles, we derive a closed expression for the transfer matrix of a linear-ramp dipole. Besides that, the minimum emittance theory is further refined, especially with more rigorous proof of the theory and parameter ranges.
\end{abstract}

DOI: 10.1103/PhysRevSTAB.15.014001

\section{INTRODUCTION}

The theoretical minimum emittance sets the emittance limit a storage ring can possibly achieve (without damping wigglers). It is of both theoretical interest and practical importance, thus was investigated over the years by many authors [1-13]. This paper is intended to be the third of our trio of papers on the theoretical minimum emittances for common lattices involving arbitrary dipole bending profiles. The first one [11] established a general formalism to compute the theoretical minimum emittance and optimal lattice parameters for a given dipole bending profile. The second one [12] used numerical optimization to investigate the optimal bending profile to yield the lowest possible emittance. This third paper analytically derives the theoretical minimum emittance based on the linear-ramped bending profile model, which consists of a short segment of constant bending radius and linear ramps at the ends, and was found to be sufficiently close to the optimal profile. Our new analytical work confirms the previous nondeterministic numerical findings in [12] and covers the whole range of potentially interesting peak field, complementary to the three isolated data points found by computation-intensive numerical optimizations. Simple practical formulas are given for determining the theoretical minimum emittance, corresponding bending profile parameters, as well as lattice parameters such as beta function

\footnotetext{
*wangcx@aps.anl.gov; http://www.aps.anl.gov/ wangcx
}

Published by the American Physical Society under the terms of the Creative Commons Attribution 3.0 License. Further distribution of this work must maintain attribution to the author(s) and the published article's title, journal citation, and DOI.
PACS numbers: 29.27.Bd, 41.75.-i, 29.20.db, 41.85.-p

and dispersion. In addition, the closed-form transfer matrix of a linear-ramp dipole is given, which is necessary for linear optics designs that involve the optimal bending profile.

Since this paper is the third of a trio, here we will skip introduction on the subject of theoretical minimum emittance in storage rings. However, in the next section, we will first present a brief review of our refined minimum emittance theory to help readers follow the new development, especially the discussions in Sec. IIB, which provides critical proofs and bounds on various parameters to make the theory more rigorous, and in Sec. II C, which clarifies some complications associated with using a reference point other than the dipole entrance for computation. Section III derives the absolute theoretical minimum emittance and simple formulas for practical applications. Sections IV and $\mathrm{V}$ present similar results for the theoretical minimum emittance in lattices requiring achromatic arcs and minimal effective emittance, respectively. Section VI gives the exact transfer matrix for dipoles with linearly changing field.

\section{MINIMUM EMITTANCE THEORY}

\section{A. Brief review of theory}

The theoretical minimum emittance with arbitrary dipoles was established [11] as

$$
\epsilon=\frac{C_{q} \gamma^{2}}{J_{x}} \mathcal{F}^{\mathrm{min}}
$$

where $C_{q}=3.84 \times 10^{-13} \mathrm{~m} ; \gamma$ is the Lorentz factor; and $J_{x}$ is the horizontal damping partition number, which we will not consider here. The lattice-dependent factor $\mathcal{F}$ is a 
function of bending profile as well as lattice type. Three commonly interested lattice types have been studied: (i) lattices with achromatic arcs, which are useful in providing dispersion-free straight sections for light sources, $\mathrm{rf}$ cavities, injection/ejection, and so on; (ii) lattices without any constraints except for minimizing the natural betatron emittance, which is the figure of merit for damping rings of linear colliders [14]; and (iii) lattices without any constraints but minimizing the effective emittance at the straight sections, which is often the figure of merit for light sources because it takes into account the effects of dispersion and beam energy spread [5]. We label these three lattice types with AME (achromatic minimum emittance), TME (theoretical minimum emittance), and EME (effective minimum emittance), respectively. The minimal $\mathcal{F}$ for these lattices reads

$$
\mathcal{F}^{\text {min }}=2 \sqrt{|E|} \begin{cases}1 & \text { TME } \\ \sqrt{1+a} & \text { AME } \\ \sqrt{\frac{[1+(q+3) q c / 2][1+\{(1+\tau) q+3] q c / 2\}}{(1-c)(1+q c)}} & \text { EME }\end{cases}
$$

where $|E|, a$, and $c=a /(a+1)$ are parameters solely determined by the dipole profile; $\tau=J_{x} / J_{E}$ is the ratio of horizontal to longitudinal damping partition numbers; and the $q$ parameter is determined by the cubic equation

$$
(1+\tau) q^{3}+2(2+\tau) q^{2}+[3+(2+\tau) / c] q+2 / c=0 .
$$

For conventional uniform dipoles of bending angle $\theta$, $2 \sqrt{|E|}=\theta^{3} / 12 \sqrt{15}, a=8$, and $c=8 / 9$ under the usually good small-angle approximation.

Equation (2) results from minimizing the following well-known expression

$$
\mathcal{F}=\frac{\left\langle\mathcal{H} /|\rho|^{3}\right\rangle}{\left\langle 1 / \rho^{2}\right\rangle} \equiv\langle\langle\mathcal{H}\rangle\rangle,
$$

where $\langle\langle\cdots\rangle$ stands for the bending-radius $(\rho)$ weighted average, and the well-known dispersion action $\mathcal{H}$ reads

$$
\begin{aligned}
\mathcal{H} & =\gamma \eta^{2}+2 \alpha \eta \eta^{\prime}+\beta \eta^{\prime 2} \\
& =\operatorname{Tr}\left\{\left(\boldsymbol{\eta}_{0} \boldsymbol{\eta}_{0}^{T}+\boldsymbol{\eta}_{0} \hat{\boldsymbol{\xi}}_{0}^{T}+\hat{\boldsymbol{\xi}}_{0} \boldsymbol{\eta}_{0}^{T}+\hat{\boldsymbol{\xi}}_{0} \hat{\boldsymbol{\xi}}_{0}^{T}\right) \sigma_{0}^{+}\right\} .
\end{aligned}
$$

The subscript 0 indicates values at the dipole entrance $s_{0}$ or reference with respect to (wrt) the entrance. $\beta, \alpha$, and $\gamma$ are the usual Courant-Snyder parameters; $\boldsymbol{\eta}_{0}=\left[\eta_{0}, \eta_{0}^{\prime}\right]^{T}$ is the initial dispersion vector; $\hat{\boldsymbol{\xi}}_{0}(s)$ is the dispersion generated in the dipole and projected back to the dipole entrance, which relates to the dispersion vector $\boldsymbol{\eta}$ via the linear transfer matrix $M$ as $\boldsymbol{\eta}(s)=M(s)\left[\boldsymbol{\eta}_{0}+\hat{\boldsymbol{\xi}}_{0}(s)\right]$; and $\sigma_{0}^{+}$ is the symplectic conjugate of the initial normalized beam matrix $\sigma_{0}$. More explicitly,

$$
\begin{aligned}
\sigma^{+} & \equiv-J \sigma^{T} J=\left[\begin{array}{ll}
\gamma & \alpha \\
\alpha & \beta
\end{array}\right], \\
\sigma & =\left[\begin{array}{cc}
\beta & -\alpha \\
-\alpha & \gamma
\end{array}\right], \\
J & =\left[\begin{array}{cc}
0 & 1 \\
-1 & 0
\end{array}\right] .
\end{aligned}
$$

Equations (4) and (5) yield

$$
\mathcal{F}=\operatorname{Tr}\left(G_{0} \sigma_{0}^{+}\right)
$$

where

$$
\left.G_{0}=\check{\rho} \boldsymbol{\eta}_{0} \boldsymbol{\eta}_{0}^{T}+\boldsymbol{\eta}_{0}\left\langle\hat{\boldsymbol{\xi}}_{0}\right\rangle{ }^{T}+\left\langle\left\langle\hat{\boldsymbol{\xi}}_{0}\right\rangle\right\rangle \boldsymbol{\eta}_{0}^{T}+\left\langle\hat{\boldsymbol{\xi}}_{0} \hat{\boldsymbol{\xi}}_{0}^{T}\right\rangle\right\rangle,
$$

and $\check{\rho} \equiv\langle\langle 1\rangle\rangle=\left\langle 1 /|\rho|^{3}\right\rangle /\left\langle 1 / \rho^{2}\right\rangle=I_{3} / I_{2} \cdot{ }^{2} I_{2}$ and $I_{3}$ are the well-known radiation integrals.

For a given dipole and initial dispersion, $G_{0}$ is determined and $\mathcal{F}$ is minimized to $2 \sqrt{\left|G_{0}\right|}$ with the optimal lattice parameters given by $\sigma_{0}=G_{0} / \sqrt{\left|G_{0}\right|}$ at the dipole entrance. From Eq. (8) it is easy to see that $\left|G_{0}\right|$ (thus $\mathcal{F}$ ) can be further minimized by choosing the initial dispersion vector along the average of the projected dispersion $\left.\left\langle\hat{\boldsymbol{\xi}}_{0}\right\rangle\right\rangle$. Let $\boldsymbol{\eta}_{0}=q\left\langle\hat{\boldsymbol{\xi}}_{0}\right\rangle / \check{\rho}$, then $G_{0}$ reduces to

$$
G=E+(q+1)^{2} \zeta \zeta^{T},
$$

where the matrix $E$ and vector $\zeta$ are given by

$$
E \equiv\left\langle\hat{\boldsymbol{\xi}} \hat{\boldsymbol{\xi}}^{T}\right\rangle-\langle\hat{\boldsymbol{\xi}}\rangle\langle\langle\hat{\boldsymbol{\xi}}\rangle\rangle^{T} / \check{\rho} \quad \text { and } \quad \zeta \equiv \frac{\langle\hat{\boldsymbol{\xi}}\rangle-\check{\rho} \hat{\boldsymbol{\xi}}\left(s_{0}\right)}{\sqrt{\check{\rho}}}
$$

We dropped the subscript 0 since these expressions are valid for any reference point, as discussed in Sec. II C. The determinant of Eq. (9) can be reduced to

$$
|G|=|E|\left[1+(q+1)^{2} a\right]
$$

where

$$
a=\operatorname{Tr}\left(E^{-1} \zeta \zeta^{T}\right)=\frac{\operatorname{Tr}\left(E^{+} \zeta \zeta^{T}\right)}{|E|}=\frac{(J \boldsymbol{\zeta})^{T} E(J \boldsymbol{\zeta})}{|E|} .
$$

The quadratic form $(J \boldsymbol{\zeta})^{T} E(J \boldsymbol{\zeta})=E_{22} \zeta_{1}^{2}-2 E_{12} \zeta_{1} \zeta_{2}+$ $E_{11} \zeta_{2}^{2}$. Note that although the matrix $E, G$, and vector $\zeta$ depend on the reference point, their determinants and the parameter $a$ do not. It can be shown that both $|E|$ and $a$ are non-negative quantities. Therefore, $\mathcal{F}^{\mathrm{min}}$ is given by $q=-1$ for TME and $q=0$ for AME lattices, as shown in Eq. (2). The derivation for EME is more involved [11].

\footnotetext{
${ }^{2}$ This was mistakenly written as $I_{2}$ instead of $I_{3} / I_{2}$ after Eq. (8) in [12]. We take this opportunity to correct a few other oversights in our previous publications. In [11], the two $\alpha_{0}$ entries in Table 1 should be switched. In both [11,12], $\hat{\xi}^{\prime}$ stands for the second component of $\hat{\boldsymbol{\xi}}$, which is not the derivative of the first component $\hat{\xi}$. To avoid confusion, we use $\hat{\xi}_{p}$ for the second component of $\hat{\boldsymbol{\xi}}$ in this paper.
} 
The expressions in Eqs. (2), (10), and (12) have the advantage of applying to any reference point. However, when using the dipole entrance as the reference point, the original expressions [11] can be more convenient for computation, especially for the AME emittance, which simply reads

$$
\mathcal{F}_{\mathrm{AME}}^{\min }=2 \sqrt{|A|}, \quad \text { where } A=\left\langle\left\langle\hat{\boldsymbol{\xi}}_{0} \hat{\boldsymbol{\xi}}_{0}^{T}\right\rangle .\right.
$$

The other drawback is that $|E|$ can approach 0 , but AME and EME emittances cannot, which is not obvious in Eq. (2). Since $|E|=|A|(1-c)$ and $|A|>0,|E|$ approaches 0 via the factor $1-c$, which is canceled by factors in the right-hand-side expressions in Eq. (2).

\section{B. Parameter ranges}

For a more rigorous theory, we show that $|E| \geq 0$, $|A|>0,|G| \geq 0, a>0$, and $0<c \leq 1$.

We have been using $|G| \geq 0$, without proof [11], to derive $\mathcal{F}^{\text {min }}=2 \sqrt{|G|}$. Here we provide the necessary proof. Since $G=E+\check{\rho}(\boldsymbol{\eta}+\langle\hat{\boldsymbol{\xi}}\rangle\rangle / \check{\rho})\left(\boldsymbol{\eta}+\langle\langle\hat{\boldsymbol{\xi}}\rangle / \check{\rho})^{T}\right.$, we will first show that $|E| \geq 0$. Consider the dot product $\odot$ of any two real integrable functions $u$ and $v$ defined by

$$
u \odot v \equiv\langle\langle u v\rangle-\langle\langle u\rangle\rangle\langle v\rangle\rangle / \check{\rho} .
$$

The linear, symmetric properties of $\odot$ are obvious. Its positive-definite property can be shown as

$$
\begin{aligned}
u \odot u= & \left\langle\left\langle u^{2}\right\rangle\right\rangle-\left\langle\langle u\rangle^{2} / \check{\rho}\right. \\
= & \frac{1}{I_{2} I_{3}}\left[\int\left(\frac{1}{|\rho|^{3 / 2}}\right)^{2} d s \int\left(\frac{u}{|\rho|^{3 / 2}}\right)^{2} d s\right. \\
& \left.-\left(\int \frac{u}{|\rho|^{3}} d s\right)^{2}\right] \geq 0,
\end{aligned}
$$

where the quantity in the square bracket is non-negative due to Cauchy-Schwarz inequality. Thus $\odot$ is a valid inner

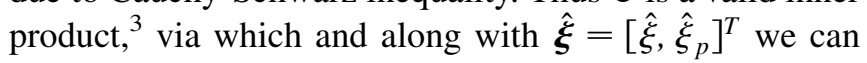
write

$$
|E|=(\hat{\xi} \odot \hat{\xi})\left(\hat{\xi}_{p} \odot \hat{\xi}_{p}\right)-\left(\hat{\xi} \odot \hat{\xi}_{p}\right)^{2} \geq 0,
$$

which is again due to Cauchy-Schwarz inequality. Note that $|A|$ can also be written in the same form with the inner product defined by $u \odot v=\langle\langle u v\rangle$. Thus $|A|>0$, unless $\hat{\xi}=\hat{\xi}_{p}$.

For a general $G$ matrix, it can be written as $G=E+$ $\boldsymbol{v} \boldsymbol{v}^{T}$ where $\boldsymbol{v}$ is some vector. If $|E|=0$, the nonnegative symmetric matrix $E$ can also be written as $\boldsymbol{u} \boldsymbol{u}^{T} ;$ therefore, $\quad G=\boldsymbol{u} \boldsymbol{u}^{T}+\boldsymbol{v} \boldsymbol{v}^{T}=[\boldsymbol{u}, \boldsymbol{v}][\boldsymbol{u}, \boldsymbol{v}]^{T}$ and $|G|=|[\boldsymbol{u}, \boldsymbol{v}]|^{2} \geq 0$. If $|E| \neq 0$, we can use $E E^{+}=|E|$ to rewrite $|G|$ as

\footnotetext{
${ }^{3}$ Rigorously speaking, $\odot$ is only a semi-inner product since it is semidefinite, i.e., there is a nonzero $u$ that yields $u \odot u=0$ (e.g., $1 \odot 1=0)$. Nonetheless, Cauchy-Schwarz inequality still holds.
}

$$
|G|=|E|\left[1+\frac{\operatorname{Tr}\left(E^{+} \boldsymbol{v} \boldsymbol{v}^{T}\right)}{|E|}\right]=|E|\left[1+\frac{\boldsymbol{v}^{T} E^{+} \boldsymbol{v}}{|E|}\right]
$$

The quadratic form $\boldsymbol{v}^{T} E^{+} \boldsymbol{v}$ is non-negative since $E$, thus $E^{+}$, is a positive-definite matrix $(|E|>0)$. Therefore $|G| \geq 0$. Setting $\boldsymbol{v}=\boldsymbol{\zeta}$, it is clear that $a>0$, unless $\zeta=0$. Furthermore, $c=a /(a+1) \in(0,1]$. (In fact, a brute force expansion of Eq. (16) yields the same result.) The upper bound is reached when $|E|=0$.

The bounds on the dipole parameter $c$ lead to bounds on the optimal $q$ parameter for minimum EME lattices. Solving the cubic equation in Eq. (3) for $c$ and imposing the bounds $0<c \leq 1$ yields $-4 / 3 \leq q_{\text {opt }}<-4 / 5$ for the nominal damping partition $\tau=1 / 2$. When $c \geq 125 / 126$, three real solutions exist. However, it can be shown that this region is not favored for minimum EME emittance lattices.

\section{Reference point}

The minimum emittance theory [11] was developed with the dipole entrance as the implied reference point. Sometimes it may be desirable to use other locations as the reference (e.g., using the center of a symmetric dipole as the reference for easier integration). The refined theory presented above applies to any reference point. Here we clarify the details.

At first glance, the original theory seems to apply to any reference point, as implied in [11]. However, this is true only for TME lattices, but not for the AME and EME lattices where the dispersion at the dipole entrance must satisfy specific conditions. Inside a dipole the dispersion vector propagates as

$$
\boldsymbol{\eta}(s)=M\left(s \mid s_{0}\right) \boldsymbol{\eta}_{0}+\boldsymbol{\xi}_{0}(s)=M\left(s \mid s_{0}\right)\left[\boldsymbol{\eta}_{0}+\hat{\boldsymbol{\xi}}_{0}(s)\right],
$$

where $\hat{\boldsymbol{\xi}}(s) \equiv M^{-1} \boldsymbol{\xi}$; the transfer matrix $M\left(s \mid s_{0}\right)$ and the dispersion-generating vector $\boldsymbol{\xi}_{0}(s)$ are determined by the dipole magnet. To use another reference point, say $s_{1}$, the dispersion can be rewritten as

$$
\begin{aligned}
\boldsymbol{\eta}(s) & =M\left(s \mid s_{1}\right)\left[\boldsymbol{\eta}_{1}-\boldsymbol{\xi}_{0}\left(s_{1}\right)\right]+\boldsymbol{\xi}_{0}(s) \\
& =M\left(s \mid s_{1}\right)\left[\boldsymbol{\eta}_{1}+\hat{\boldsymbol{\xi}}_{1}(s)\right],
\end{aligned}
$$

with

$$
\hat{\boldsymbol{\xi}}_{1}(s)=M\left(s_{1} \mid s_{0}\right) \hat{\boldsymbol{\xi}}_{0}(s)-\boldsymbol{\xi}_{0}\left(s_{1}\right)
$$

Note that $\hat{\boldsymbol{\xi}}_{0}\left(s_{0}\right)=0$ and thus $\boldsymbol{\xi}_{0}\left(s_{1}\right)=-\hat{\boldsymbol{\xi}}_{1}\left(s_{0}\right)$. Using the average of Eq. (20), the dispersion at $s_{1}$ resulting from $\boldsymbol{\eta}_{0}=q\left\langle\left\langle\hat{\boldsymbol{\xi}}_{0}\right\rangle / \check{\rho}\right.$ can be written as

$$
\begin{aligned}
\boldsymbol{\eta}_{1} & =M\left(s_{1} \mid s_{0}\right)\left(q\left\langle\left\langle\hat{\boldsymbol{\xi}}_{0}\right\rangle / \check{\boldsymbol{\rho}}\right)+\boldsymbol{\xi}_{0}\left(s_{1}\right)\right. \\
& =q\left\langle\left\langle\hat{\boldsymbol{\xi}}_{1}\right\rangle / \check{\boldsymbol{\rho}}-(q+1) \hat{\boldsymbol{\xi}}_{1}\left(s_{0}\right) .\right.
\end{aligned}
$$


Inserting $\boldsymbol{\eta}_{1}$ into $G_{1} \equiv \check{\rho} \boldsymbol{\eta}_{1} \boldsymbol{\eta}_{1}^{T}+\boldsymbol{\eta}_{1}\left\langle\left\langle\hat{\boldsymbol{\xi}}_{1}\right\rangle{ }^{T}+\left\langle\hat{\boldsymbol{\xi}}_{1}\right\rangle\right\rangle \boldsymbol{\eta}_{1}^{T}+$ $\left\langle\hat{\boldsymbol{\xi}}_{1} \hat{\boldsymbol{\xi}}_{1}^{T}\right\rangle$, after some algebra, we end up with the reference-point-covariant form in Eq. (9). Note that one cannot set $\boldsymbol{\eta}_{1}=q\left\langle\left\langle\hat{\boldsymbol{\xi}}_{1}\right\rangle\right\rangle / \check{\rho}$, which is true only for TME lattices with $q=-1$ or for $\boldsymbol{\xi}_{0}\left(s_{1}\right)=0$, which means no bending between the two reference points.

Note that the projected dispersion-generating vector $\hat{\boldsymbol{\xi}}$ wrt any reference point $s_{1}$ can be computed directly using

$$
\hat{\boldsymbol{\xi}}^{\prime}=\left[\begin{array}{c}
\hat{\boldsymbol{\xi}}^{\prime} \\
\hat{\xi}_{p}^{\prime}
\end{array}\right]=M^{-1}\left[\begin{array}{c}
0 \\
1 / \rho
\end{array}\right], \quad \hat{\boldsymbol{\xi}}\left(s_{1}\right)=0,
$$

where $M$ is the transfer matrix starting from $s_{1}$.

\section{THEORETICAL MINIMUM EMITTANCE}

In this section we analytically derive the theoretical minimum emittance (TME) using the linear-ramp model with bending radius $\rho$ given by

$\rho(s)=\rho_{0} \times \begin{cases}1 & |s| \leq L_{0} \\ 1+g\left(|s| / L_{0}-1\right) & L_{0} \leq|s| \leq L,\end{cases}$

where $g=(r-1) /\left(L / L_{0}-1\right)$ and $r \equiv \rho_{\max } / \rho_{0}$. This symmetric dipole extends from $-L$ to $L$ with a uniform central segment from $-L_{0}$ to $L_{0}$ and linear ramps at the ends. This profile is of particular interest because it is sufficiently simple for analytical treatment yet has been shown to be very close to the optimal profile for TME emittance, i.e., the absolute theoretical minimum.

The bending angle $\theta(s)=\int_{0}^{s} d s / \rho$ is given by

$$
\theta(s)= \begin{cases}\frac{s}{\rho_{0}} & |s| \leq L_{0} \\ \operatorname{sgn}(s) \frac{L_{0}}{\rho_{0}}\left(1+\frac{1}{g} \ln \frac{\rho}{\rho_{0}}\right) & L_{0} \leq|s| \leq L,\end{cases}
$$

and half of the total bending angle is given by

$$
\theta_{\max }=\theta_{0}(1+\ln r / g)
$$

where $\theta_{0}=L_{0} / \rho_{0}$ is the angle contribution from the central constant field. The ratio of maximum dipole field strength to that of the equivalent uniform dipole with the same length and bending angle reads

$$
\kappa \equiv \frac{B_{\max }}{B_{\text {ref }}}=\frac{\rho_{\text {ref }}}{\rho_{\min }}=\frac{L}{L_{0}} \frac{\theta_{0}}{\theta_{\max }}=\frac{g+r-1}{g+\ln r} .
$$

Since the emittance can potentially reach zero as $\kappa \rightarrow \infty$, we choose $\kappa$ as a given parameter and optimize $r \in[1, \infty]$ for the minimum emittance. The other parameters can be rewritten as

$$
\frac{L_{0}}{L}=\frac{r-1-\kappa \ln r}{\kappa(r-1-\ln r)}, \quad \frac{\theta_{0}}{\theta_{\max }}=\frac{L_{0}}{L} \kappa,
$$

and

$$
g=\frac{r-1-\kappa \ln r}{\kappa-1}
$$

Since $r-1-\ln r \geq 0, L_{0} / L>0$ implies a minimum $r$ for a given $\kappa$, which is the solution of $r-1-\kappa \ln r=0$, i.e., $r_{\min }=-\kappa W\left(-e^{-1 / \kappa} / \kappa\right)$, where $W$ is the Lambert $W$ function.

For computing the TME emittance and optimal lattice parameters, we take advantage of the symmetry and use the dipole center as the reference. For simplicity, we ignore the weak focusing effects of the dipole ${ }^{4}$ and use Eq. (22) with $M=M_{\text {drfit }}$ to compute $\hat{\boldsymbol{\xi}}$, which yields $\hat{\xi}_{p}=\int_{0}^{s} d s / \rho=$ $\theta(s)$ and $\hat{\xi}=-\int_{0}^{s} s d s / \rho$, i.e.,

$\hat{\xi}= \begin{cases}-s^{2} / 2 \rho_{0} & |s| \leq L_{0} \\ -\frac{L_{0}^{2}}{2 \rho_{0}}\left\{1+\frac{2}{g^{2}}\left[(g-1) \ln \frac{\rho}{\rho_{0}}+\frac{\rho}{\rho_{0}}-1\right]\right\} & L_{0} \leq|s| \leq L .\end{cases}$

Inserting $\hat{\boldsymbol{\xi}}$ into Eq. (10), we can compute the matrix $E$ as well as emittance and Twiss parameters at the dipole center. Thanks to the symmetry, $\hat{\xi}$ is an even function and $\hat{\xi}_{p}$ is an odd function, thus $E$ reduces to a simple diagonal matrix

$$
E=\operatorname{diag}\left(\left\langle\hat{\xi}^{2}\right\rangle\right\rangle-\left\langle\langle\hat{\xi}\rangle^{2} / \check{\rho},\left\langle\hat{\xi}_{p}^{2}\right\rangle\right) .
$$

Carrying out the straightforward integrations (see Appendix A), we have

$$
E_{11}=\frac{L_{0}^{4}}{\rho_{0}^{3}} \frac{f_{1}}{360 g^{4} r[(g+1) r-1]\left[(2 g+1) r^{2}-1\right]}
$$

and

$$
E_{22}=\frac{L_{0}^{2}}{\rho_{0}^{3}} \frac{f_{2}}{12 g^{2} r(g r+r-1)},
$$

where

$$
\begin{aligned}
f_{1}= & 16 g^{6} r^{4}+24 g^{4}(2 g+5) r^{2}\left(r^{2}-1\right) \\
& +60 g^{3} r^{2}(r-1)(5 r-3)+45 g^{2}(r-1)^{2}\left(17 r^{2}+2 r+1\right) \\
& -90 g(r-1)\left(19 r^{3}-9 r^{2}+3 r-1\right) \\
& -45(r-1)^{2}\left(23 r^{2}+6 r-1\right) \\
& -180(r \ln r)^{2}(g-1)^{2}(2 g+1)-120 r^{2} \ln r\left[g^{3}+2 g^{4}\right. \\
& \left.+3(r-1)\left(4 g^{2}-r-3\right)-3 g\left(2 r^{2}+2 r-1\right)\right]
\end{aligned}
$$

and

\footnotetext{
${ }^{4}$ Focusing in uniform (including combined-function) dipoles was found to have small higher-order effect on the minimum emittance [11], which is probably true for variable bending as well, although the field can be much stronger (but shorter). Combined-function dipoles could be more useful in terms of manipulating partition numbers, implementing a low-beta lattice, and designing more compact lattices.
} 


$$
\begin{aligned}
f_{2}= & 4 g^{3} r^{2}+3\left(2 g^{2}+2 g+1\right)\left(r^{2}-1\right) \\
& -6 \ln r(2 g+1+\ln r)
\end{aligned}
$$

The TME emittance is simply $2 \sqrt{E_{11} E_{22}}$, which reads

$$
\mathcal{F}^{\mathrm{TME}}=\frac{\theta_{0}^{3}}{6 g^{3} r[(g+1) r-1]} \sqrt{\frac{f_{1} f_{2}}{30\left[(2 g+1) r^{2}-1\right]}}
$$

Normalizing this expression by the TME emittance of a uniform dipole with the same bending angle, which is $\theta^{3} / 12 \sqrt{15}=8 \theta_{\max }^{3} / 12 \sqrt{15}=(2 / 3 \sqrt{15})\left[\theta_{0}(1+\ln r / g)\right]^{3}$, we obtain the emittance reduction factor

$$
\hat{\mathcal{F}}^{\mathrm{TME}}=\frac{1}{r(g+\ln r)^{3}[(g+1) r-1]} \sqrt{\frac{f_{1} f_{2}}{32\left[(2 g+1) r^{2}-1\right]}} .
$$

It is easy to see that as $r \rightarrow 1, \hat{\mathcal{F}}^{\mathrm{TME}} \rightarrow 1$, as it should reduce to the uniform dipole. It is not so obvious but confirmed that, as $L_{0} \rightarrow 0$, Eq. (34) reduces to the result of the symmetric-linearly increasing profile given in the Appendix of [12]. Note that as $L_{0} \rightarrow 0, g \rightarrow 0$ and $L_{0} / g \rightarrow$ $L /(r-1)$. It can also be shown that $\hat{\mathcal{F}}^{\mathrm{TME}} \rightarrow 0$ as $\kappa \rightarrow \infty$, which is why $\kappa$ has to be given.

Inserting Eq. (28) into Eq. (34) we obtain $\hat{\mathcal{F}}^{\mathrm{TME}}(\kappa, r)$ whose inverse is shown in Fig. 1. The existence of optimal profiles is evident and can be determined by numerically solving the equation $d \hat{\mathcal{F}} / d r=0$ for a given $\kappa$. The resulting absolute minimum emittance and the required optimal $r_{\text {opt }}$ are plotted in Figs. 2 and 3, respectively. Simple approximate formulas are also given in the figure captions for practical applications.

The optimal Twiss parameters at the dipole center are $\alpha_{c}=0$ and $\beta_{c}=\sqrt{E_{11} / E_{22}}$, which is

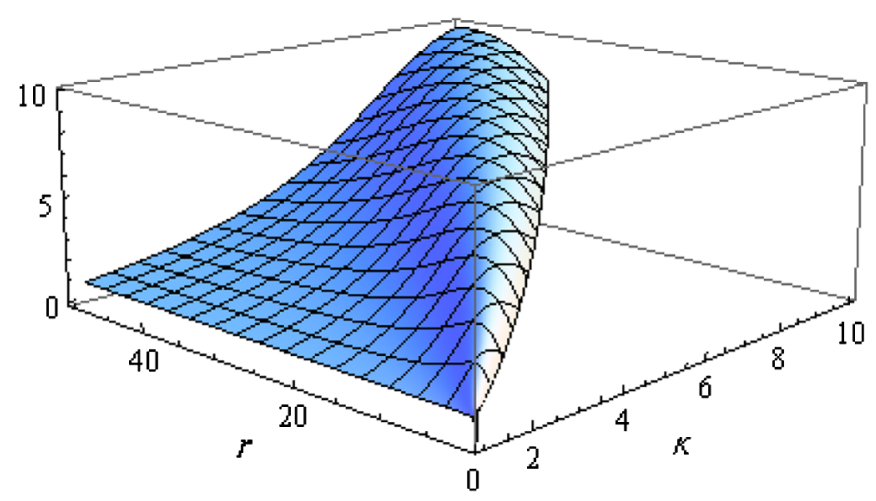

FIG. 1. TME emittance improvement factor $1 / \hat{\mathcal{F}}^{\mathrm{TME}}$ as a function of $\kappa=B_{\max } / B_{\text {ref }}$ and $r=\rho_{\max } / \rho_{0}$. The obvious ridge indicates the maximum emittance reduction.

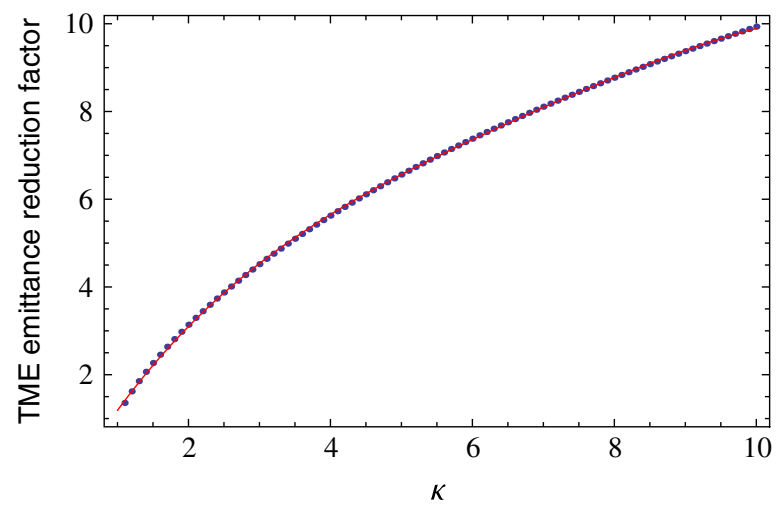

FIG. 2. Maximum TME emittance improvement factor as a function of $\kappa=B_{\max } / B_{\text {ref }}$. The blue dots are the exact values. The red curve is given by the approximate expression $1 / \hat{F}(\kappa)=$ $-1.389+2.981 \kappa-0.4428 \kappa^{2}+0.04012 \kappa^{3}-0.001437 \kappa^{4}$. The values at $\kappa=2,4$, and 6 agree with the results in [12].

$$
\frac{\beta_{c}}{L_{\text {dipole }}}=\frac{1}{g+r-1} \sqrt{\frac{f_{1} / f_{2}}{120\left[(2 g+1) r^{2}-1\right]}},
$$

where $L_{\text {dipole }}=2 L$ is the total length of the dipole. The optimal $\beta_{c} / L_{\text {dipole }}$ is plotted in Fig. 4 . Note that the required small beta function represents the intrinsic challenge for implementing minimum emittance lattice in practice. We will comment more on this point later.

The dispersion reaches a minimum at the dipole center, whose value is given by $\eta_{c}=-\langle\langle\hat{\xi}\rangle / \check{\rho}$, i.e.,

$$
\frac{\eta_{c}}{L_{\text {dipole }} \theta}=\frac{2 g^{3}+3\left[1+g+g^{2}-\frac{4}{r}+\frac{3-g-g^{2}+2(1-g) \ln r}{r^{2}}\right]}{24(g+\ln r)(g+r-1)\left(2 g+1-1 / r^{2}\right)},
$$

where $\theta$ is the total bending angle. Figure 5 plots the factor $\eta_{c} / \theta / L_{\text {dipole }}$ for the minimum TME lattice.

To close our discussion on the TME emittance, we consider the increase of beam energy spread due to bending profile variation. The enlargement factor is given by

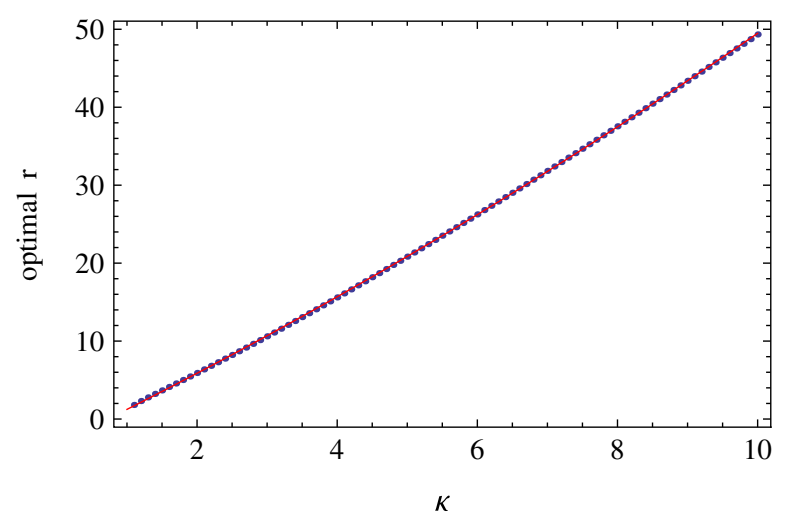

FIG. 3. The optimal bending profile parameter $r_{\mathrm{opt}}=\rho_{\mathrm{max}} / \rho_{0}$ as a function of $\kappa$. The red curve is given by the approximate expression $r_{\text {opt }}(\kappa)=-3.21+4.36 \kappa+0.0909 \kappa^{2}$. 


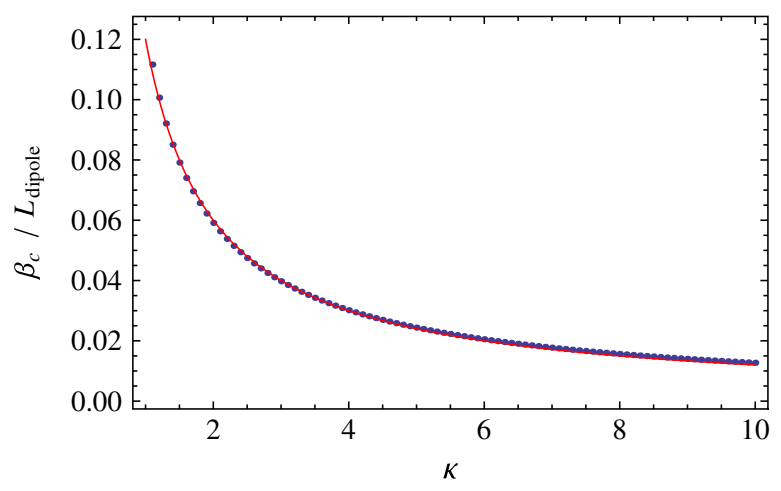

FIG. 4. The beta minimum over dipole length for the absolute minimum emittance. The red curve is given by the approximate expression $\beta / L_{\text {dipole }}=0.12 / \kappa$.

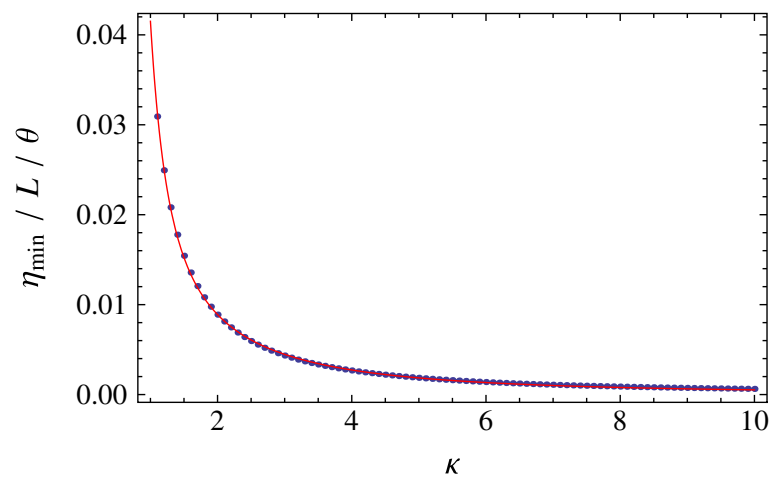

FIG. 5. The dispersion minimum over dipole length and bending angle for the absolute minimum emittance. The red curve is given by $\eta / \theta / L_{\text {dipole }}=0.059 / \kappa^{2}-0.077 / \kappa^{3}+0.059 / \kappa^{4}$.

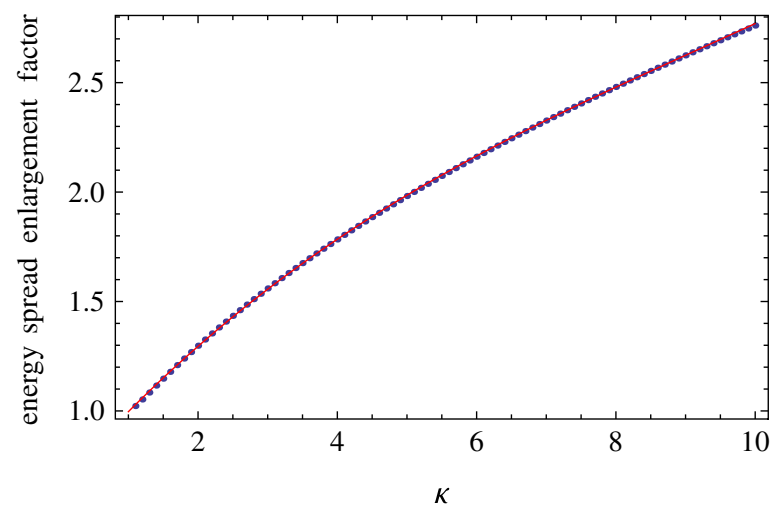

FIG. 6. Beam energy-spread enlargement factor $\left(\sigma_{E}\right.$ over $\sigma_{E}$ of the reference uniform dipole) for the absolute minimum emittance lattice. The red curve is given by the approximate expression $0.655+0.365 \kappa-0.0242 \kappa^{2}+0.000885 \kappa^{3}$.

$$
\sqrt{\frac{\check{\rho}}{1 / \rho_{\text {ref }}}}=\sqrt{\frac{g+r-1}{g+\ln r} \frac{g+\left(1-1 / r^{2}\right) / 2}{g+1-1 / r}},
$$

which is plotted in Fig. 6 under the minimum TME conditions.

\section{MINIMUM AME EMITTANCE}

Because of the zero dispersion requirement at the dipole entrance (or exit), AME lattices require a different optimal bending profile from that of TME lattices. It is asymmetric and consists of a constant field followed by a linear ramp. In other words, it basically is one-half of the TME profile. Thus, much the computation for the TME profiles can be used for AME emittance computation. In the above TME computation, the profile extends from $-L$ to $L$, and the center was used as the reference point. For AME computation, we can simply use the same profile and various integrals but limit to $s \geq 0$ and use the dipole entrance as the reference point. The major difference is that, due to the asymmetry, $\left\langle\hat{\xi} \hat{\xi} \hat{\xi}_{p}\right\rangle$ is not zero anymore and needs to be computed to obtain the minimum AME emittance $2 \sqrt{|A|}$. The results reads

$$
\hat{\mathcal{F}}^{\mathrm{AME}}=\frac{\sqrt{f_{3}}}{r(g+\ln r)^{3}[(g+1) r-1]},
$$

where

$$
\begin{aligned}
f_{3}= & g^{8} r^{4}+2 g^{6}(2 g+7) r^{2}\left(r^{2}-1\right)+4 g^{3}\left[g^{2} r^{2}(13 r-7)\right. \\
& \left.+5\left(35 r^{3}-17 r^{2}+9 r-3\right)\right](r-1) \\
& +5\left[g^{4}\left(43 r^{2}+6 r+3\right)+24^{2} g^{2} r^{2}\right. \\
& \left.+24 g(g-1)\left(13 r^{2}-2 r+1\right)-12\left(15 r^{2}-2 r-1\right)\right] \\
& \times(r-1)^{2}-4 r \ln r\left\{(7 g+6) g^{5} r+100 g^{4} r(r-1)\right. \\
& -40 g^{3} r^{2} \times(2 r+1)-60(r-1)\left[g^{2}\left(2 r^{2}+5 r-1\right)\right. \\
& \left.\left.+2 g\left(r^{2}+4 r+1\right)+r^{2}+7 r-2\right]\right\} \\
& +4(r \ln r)^{2}\left(16 g^{5}-35 g^{4}-20 g^{3}\right. \\
& +360 g+180)-480 r^{2}(\ln r)^{3} .
\end{aligned}
$$

Again, it is easy to see that $\hat{\mathcal{F}}^{\mathrm{AME}} \rightarrow 1$ as $r \rightarrow 1$. As $L_{0} \rightarrow 0$ it reduces to the result of the linearly increasing profile given in the Appendix of [12].

The parametric plot of the AME emittance improvement factor $1 / \hat{\mathcal{F}}^{\mathrm{AME}}$ is similar to Fig. 1 with a flatter ridge. The maximum enhancement factor for AME emittance and the corresponding optimal $r_{\text {opt }}$ are plotted in Figs. 7 and 8. We see that the AME improvement factor is about half of the TME improvement factor.

The Twiss parameters at the dipole entrance are given by $\beta_{0}=A_{11} / \sqrt{|A|}$ and $\alpha_{0}=-A_{12} / \sqrt{|A|}$, which yields 


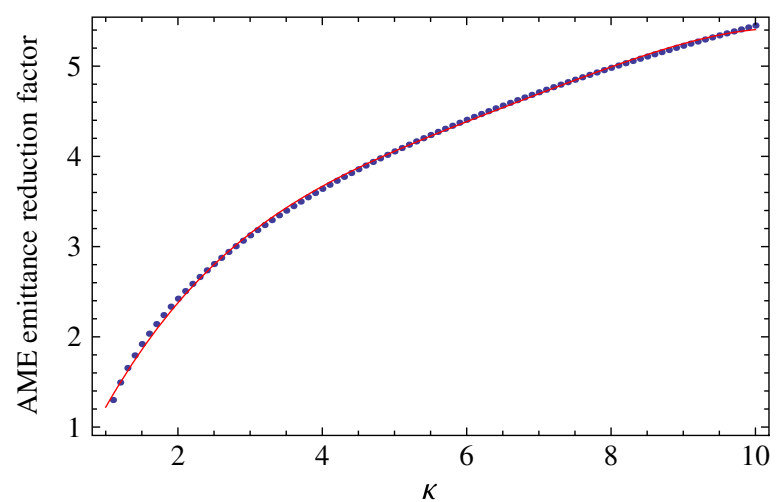

FIG. 7. Maximum improvement factor for AME emittance as a function of $\kappa=B_{\max } / B_{\text {ref }}$. The blue dots are the exact values. The red curve is given by the approximate expression $1 / \hat{F}(\kappa)=$ $-0.504+2.08 \kappa-0.389 \kappa^{2}+0.0383 \kappa^{3}-0.00143 \kappa^{4}$. The values at $\kappa=2,4$, and 6 agree with the results in [12].

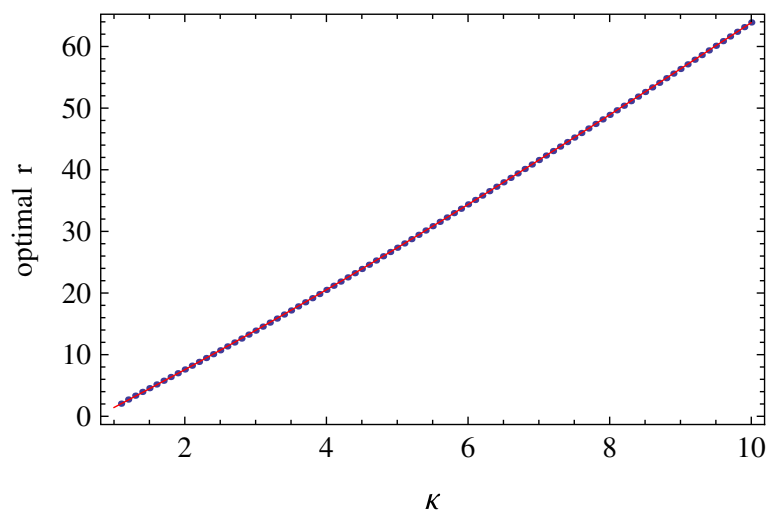

FIG. 8. The optimal bending profile parameter $r_{\mathrm{opt}}=\rho_{\mathrm{max}} / \rho_{0}$ as a function of $\kappa$ for minimum AME emittance. The red curve is given by the approximate expression $r_{\mathrm{opt}}(\kappa)=-4.49+$ $5.81 \kappa+0.101 \kappa^{2}+0.00379 \kappa^{3}-0.000364 \kappa^{4}$.

$$
\begin{aligned}
\frac{\beta_{0}}{L}= & \frac{6}{\sqrt{15}} \\
& \times \frac{2 g^{5}+(r-1) z_{1}-20 \ln r z_{2}+20(g-1)^{2}(\ln r)^{2}}{2(g+r-1) \sqrt{f_{3}}},
\end{aligned}
$$

where $\quad z_{1}=50-40 g-20 g^{2}+10 g^{3}+5 g^{4}+2 g^{5}+$ $\left(-110+40 g+20 g^{2}+10 g^{3}+5 g^{4}+2 g^{5}\right) r \quad$ and $\quad z_{2}=$ $g^{3}+3+4 g(r-1)-2 r(r+2)$, and

$\alpha_{0}=\sqrt{15} \frac{g^{4} r^{2}+4(g+1)(r-1)^{2}+g^{2}(3+2 g)\left(r^{2}-1\right)}{\sqrt{f_{3}}}$.

The optimal values of $\beta_{0} / L$ and $\alpha_{0}$ are plotted in Figs. 9 and 10. Ignoring focusing, the corresponding minimum beta is given by $\beta_{\min }=1 / \gamma_{0}=\beta_{0} /\left(1+\alpha_{0}^{2}\right)$. Furthermore, the phase advance $\Delta \psi$ across the dipole is given by

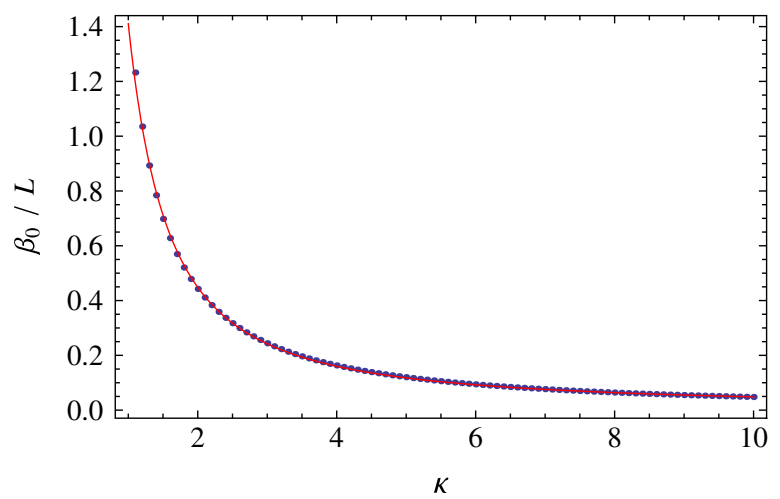

FIG. 9. The initial beta over dipole length for the minimum AME emittance. The red curve is given by the approximate expression $\beta_{0} / L_{\text {dipole }}=0.383 / \kappa+1.03 / \kappa^{2}$.

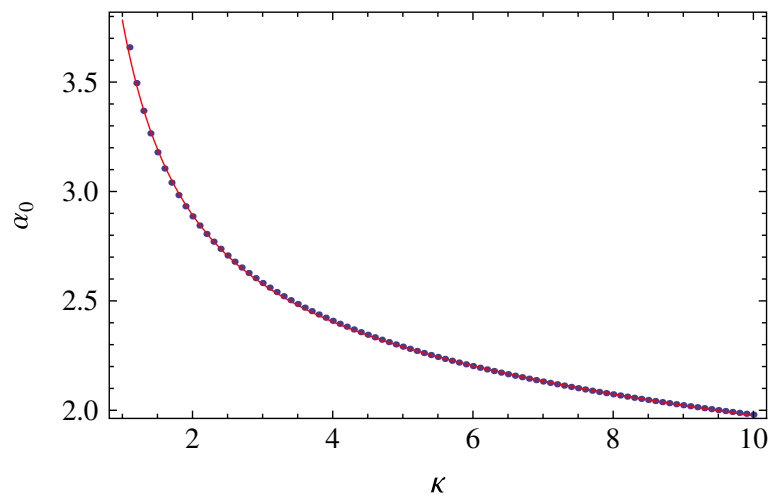

FIG. 10. The initial $\alpha$ for the minimum AME emittance. The red curve is given by the approximate expression $\alpha_{0}=$ $2.09+1.72 / \kappa-0.029 \kappa$.

$$
\Delta \psi=\tan ^{-1}\left(\gamma_{0} L-\alpha_{0}\right)+\tan ^{-1} \alpha_{0} .
$$

The energy-spread enlargement factor for AME lattices is still given by Eq. (37), but the optimal $r$ value is somewhat different, which yields a slightly different energyspread enlargement factor given by the approximate expression $0.72+0.32 \kappa-0.011 \kappa^{2}$.

\section{MINIMUM EME EMITTANCE}

The optimal bending profile for minimum EME emittance is more complicated. Available numerical optimizations [12] suggest an asymmetric bending profile starting with a drift, then a fast linear ramp in magnetic field leading to the main constant bending field, followed by a linear ramp in bending radius to the end of the dipole. If we ignore the short segment of linear ramp in magnetic field, the profile is the same as the optimal AME profile except for the initial drift, which yields a larger reference bending radius $\rho_{\text {ref }}$ and thus higher $\kappa$ for the same performance. Thus, the optimal profile should have no drift, contradicting the numerical optimization. To resolve this, further 
investigation is needed to refine both the simulation with an increased number of slices and the analytical computation by taking the linear magnetic field ramp into account. However, since the emittance appears insensitive to such details, we consider only a constant field followed by a linear ramp in bending radius for minimum EME emittance computation, i.e., the same profile as in the AME emittance but with different parameters.

To compute the EME emittance, using the dipole entrance as the reference, we need to compute the parameter $c=-\operatorname{Tr}[J A J B] /|A|$ with $A=\left\langle\left\langle\hat{\boldsymbol{\xi}}_{0} \hat{\boldsymbol{\xi}}_{0}^{T}\right\rangle\right.$ and $B=$ $\left.\left\langle\left\langle\hat{\boldsymbol{\xi}}_{0}\right\rangle\right\rangle\left\langle\hat{\boldsymbol{\xi}}_{0}\right\rangle\right\rangle^{T} / \check{\rho}$, which gives

$c=\frac{16 g^{9} r^{6}+\left(r^{2}-1\right) c_{1}+(r-1)^{2} c_{2}+(r-1) c_{3}+c_{4} \ln r}{9\left[(2 g+1) r^{2}-1\right] f_{3}}$,

where $c_{1}$ to $c_{4}$ are messy polynomials of $g$ and $r$ that are found in Appendix B. Clearly $c$ reduces to the uniform dipole value $8 / 9$ as $r \rightarrow 1$.

Using the expression for $c$, we solve the cubic equation in Eq. (3) for the optimal dispersion parameter $q$, assuming the common damping partition $\tau=1 / 2$. Then the minimum EME emittance can be determined by Eq. (2). The parametric plot of the EME emittance improvement factor $1 / \hat{\mathcal{F}}^{\mathrm{EME}}$ is again similar to Fig. 1 . The maximum EME emittance improvement as a function of $\kappa$ is plotted in Fig. 11, while the optimal $r$ parameter is plotted in Fig. 12.

The optimal Twiss parameters can be computed via $\sigma=$ $G / \sqrt{|G|}$ with $G$ given by Eq. (9). We skip the messy analytical result and simply plot the optimal initial $\beta_{0} / L$ and $\alpha_{0}$ in Figs. 13 and 14. Again, $\beta_{\min }=1 / \gamma_{0}$, and the phase advance across the dipole is given by Eq. (41).

The optimal dispersion at the dipole entrance is given by $\boldsymbol{\eta}_{\mathrm{opt}}=q_{\mathrm{opt}}\left\langle\left\langle\hat{\boldsymbol{\xi}}_{0}\right\rangle / / \check{\rho}\right.$, which yields

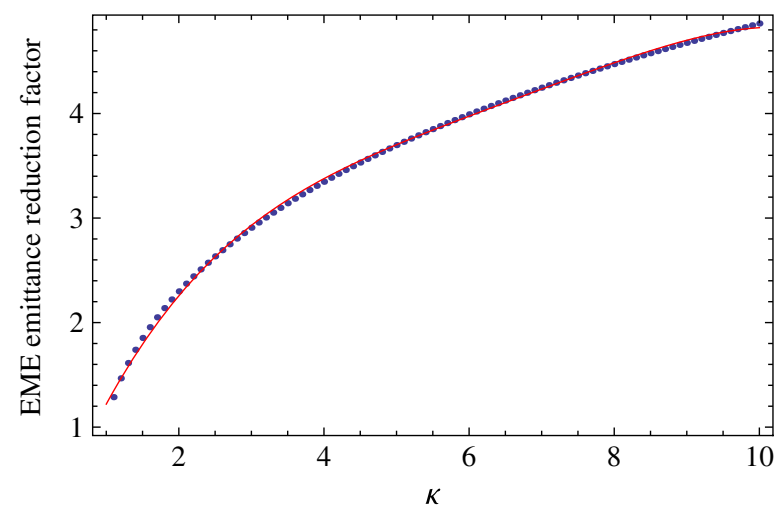

FIG. 11. Maximum improvement factor for EME emittance as a function of $\kappa=B_{\max } / B_{\text {ref }}$. The blue dots are the exact values. The red curve is given by the approximate expression $1 / \hat{F}(\kappa)=$ $-0.349+1.9 \kappa-0.366 \kappa^{2}+0.0364 \kappa^{3}-0.00137 \kappa^{4}$. The values at $\kappa=2,4$, and 6 agree with the results in [12].

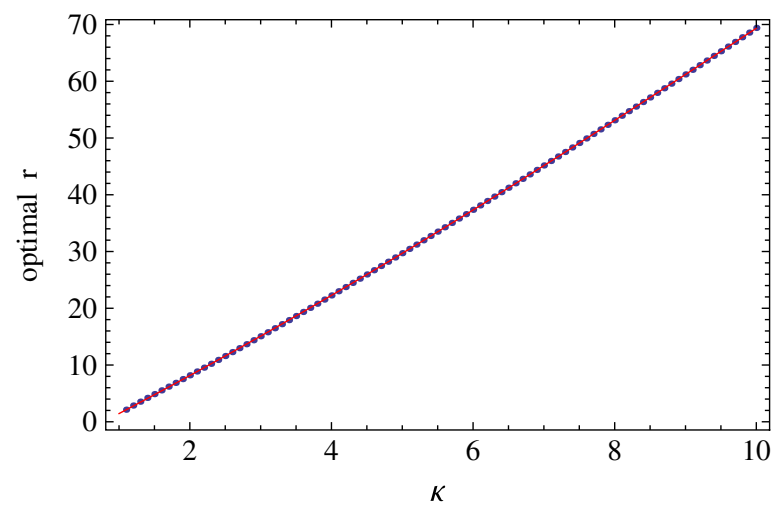

FIG. 12. The optimal bending profile parameter $r_{\mathrm{opt}}=$ $\rho_{\max } / \rho_{0}$ as a function of $\kappa$ for minimum EME emittance. The red curve is given by the approximate expression $r_{\mathrm{opt}}(\kappa)=$ $-5.03+6.37 \kappa+0.103 \kappa^{2}+0.00426 \kappa^{3}-0.000389 \kappa^{4}$.

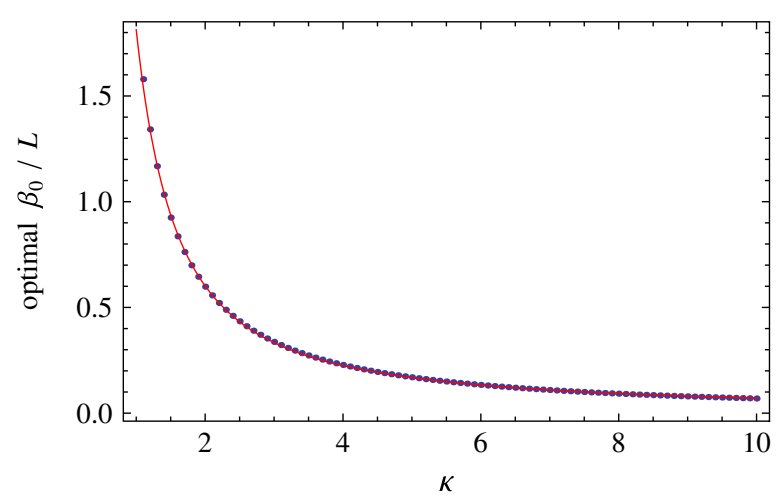

FIG. 13. The initial beta over dipole length for the minimum EME emittance. The red curve is given by the approximate expression $\beta_{0} / L_{\text {dipole }}=0.593 / \kappa+1.22 / \kappa^{2}$.

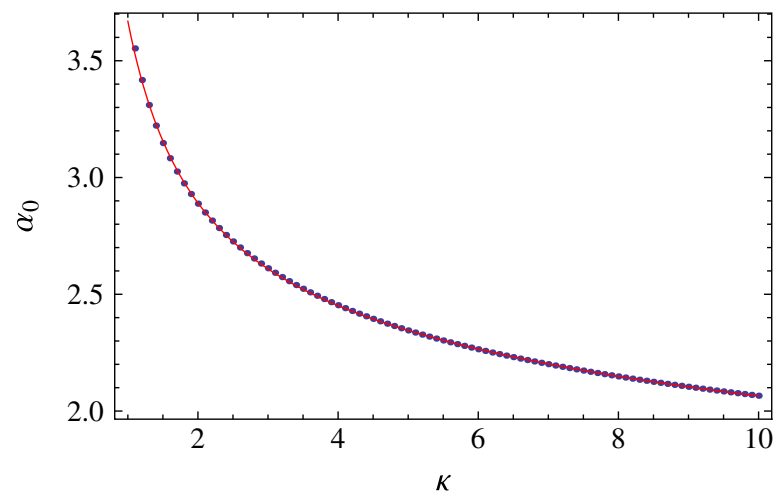

FIG. 14. The initial $\alpha_{0}$ for the minimum EME emittance. The red curve is given by the approximate expression $\alpha_{0}=2.23+$ $1.48 / \kappa-0.04 \kappa$. 


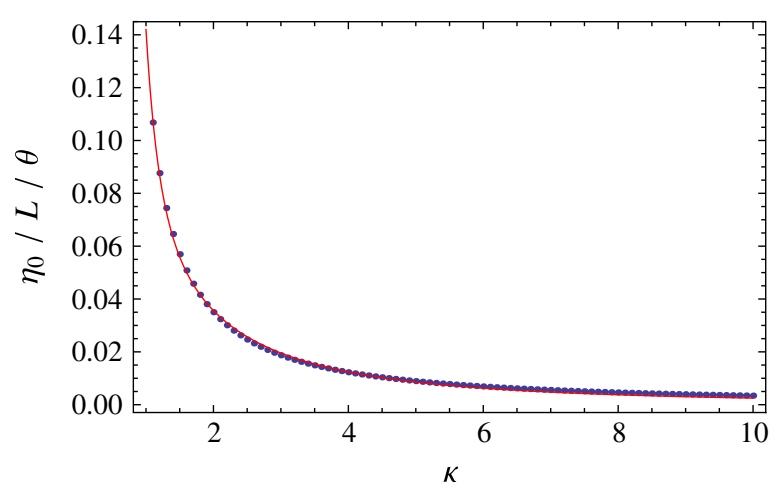

FIG. 15. Initial dispersion $\eta_{0} /(L \theta)$ for minimum EME emittance. The red curve is given by the approximate expression $0.298 / \kappa^{4}-0.448 / \kappa^{3}+0.292 / \kappa^{2}$.

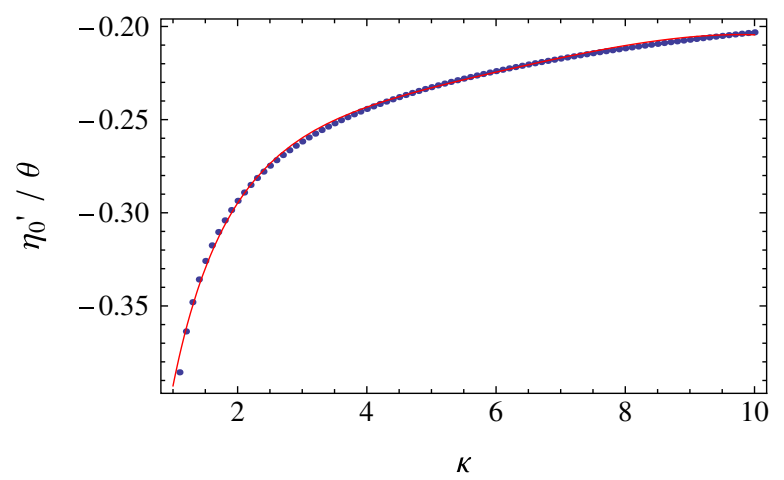

FIG. 16. Initial dispersion slope $\eta_{0}^{\prime} / \theta$ for minimum EME emittance. The red curve is given by the approximate expression $-0.281-0.122 \kappa+0.0105 \kappa^{2}-0.000389 \kappa^{3}+0.276 \ln [k]$.

$$
\frac{\eta_{0}}{q_{\mathrm{opt}} L \theta}=-\frac{2 g^{3}+3\left[1+g+g^{2}-\frac{4}{r}+\frac{3-g-g^{2}+2(1-g) \ln r}{r^{2}}\right]}{6(g+\ln r)(g+r-1)\left(2 g+1-1 / r^{2}\right)}
$$

and

$$
\frac{\eta_{0}^{\prime}}{q_{\mathrm{opt}} \theta}=\frac{2 g^{2}+2 g+1-(2 g+1+2 \ln r) / r^{2}}{2(g+\ln r)\left(2 g+1-1 / r^{2}\right)} .
$$

Note that, although the right-hand side of Eq. (43) has the same expression as that of Eq. (36) except for a -4 factor, the optimal profile parameters $g$ and $r$ are different for TME and EME lattices. Figures 15 and 16 plot the optimal values of initial dispersion for minimum EME lattices.

\section{TRANSFER MATRIX OF A LINEAR-RAMP DIPOLE}

Transfer matrices are essential for lattice designs. To facilitate investigation of lattices using the optimal dipole profile with linear ramps, we present the exact transfer matrix of a linear-ramp dipole whose bending radius changes linearly inside the dipole. What needs to be solved is the dispersion equation

$$
\frac{d^{2} D}{d s^{2}}+\frac{1}{\rho(s)^{2}} D=\frac{1}{\rho(s)}
$$

with $\rho(s)=\rho_{0}+\rho^{\prime} s$, and the initial condition $D_{0}=$ $D_{0}^{\prime}=0$ at the entrance.

An obvious special solution of this inhomogeneous differential equation is $D=\rho$. The general solution is a summation of this special solution with the general solution of the corresponding homogenous equation. It is interesting to note that this homogeneous equation is the same as the equation of motion (in the reduced coordinates) for a particle passing an accelerating rf cavity, as discussed in Appendix D of [15]. The solution is given by the transfer matrix

$$
M=\left[\begin{array}{cc}
\sqrt{\frac{\rho}{\rho_{0}}}\left(\cos u-\frac{1}{2 \varpi} \sin u\right) & \frac{\sqrt{\rho_{0} \rho}}{\varpi \rho^{\prime}} \sin u \\
-\frac{\varpi \rho^{\prime}}{\sqrt{\rho_{0} \rho}}\left(1+\frac{1}{4 \varpi^{2}}\right) \sin u & \sqrt{\frac{\rho_{0}}{\rho}\left(\cos u+\frac{1}{2 \varpi} \sin u\right)}
\end{array}\right],
$$

where $u \equiv \varpi \ln \frac{\rho}{\rho_{0}}$ and $\varpi \equiv \sqrt{\frac{1}{\rho^{2}}-\frac{1}{4}}$. To get the dispersiongenerating vector $\boldsymbol{\xi}$, we only need to pick the special solution with the initial condition $\boldsymbol{\xi}(0)=0$, which is $\boldsymbol{\xi}=$ $\left[\rho, \rho^{\prime}\right]^{T}-M\left[\rho_{0}, \rho^{\prime}\right]^{T}$ and reads

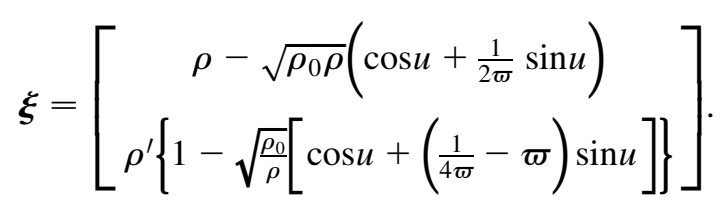

In case $\rho^{\prime}=2$, one has to take the limit $\varpi \rightarrow 0$ for all elements in $M$ and $\boldsymbol{\xi}$ given above.

\section{CONCLUDING REMARKS}

Based on simple linear-ramped bending profiles that have been shown sufficiently close to the optimal for reaching the minimum emittance, we computed the theoretical minimum emittance for TME, AME, and EME lattices with arbitrary bending profiles. For TME lattices, the profile is symmetric with a constant field segment at the center and linear ramps at both ends. For AME and EME lattices it is asymmetric, starting with a constant field segment followed by a linear ramp to the end. Suppose the constant field is $\kappa$ times the reference uniform dipole field, the optimal lengths of this constant segment for TME, AME, and EME as a fraction of the dipole length is given by Eq. (27) and are plotted in Fig. 17. The corresponding theoretical-minimum-emittance improvement factors over the reference dipole are summarized in Fig. 18. Analytical formulas as well as approximate expressions are given for the theoretical minimum emittances and required optics parameters. 


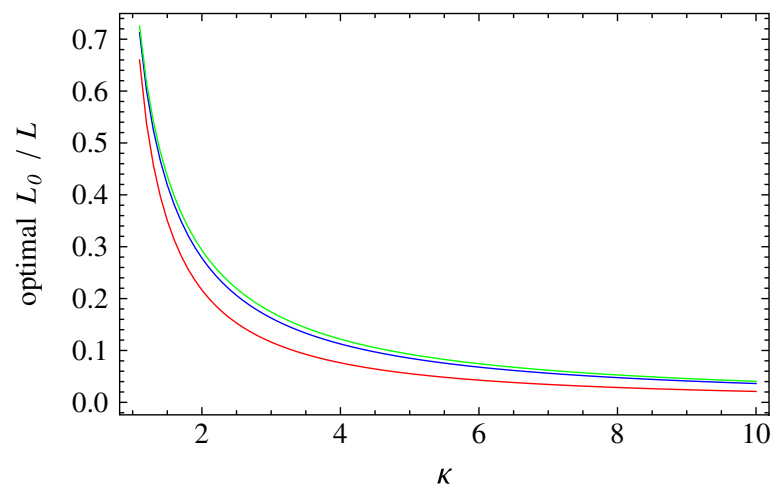

FIG. 17. Parameters of optimal linear-ramped bending profiles. The constant peak field segment is $\kappa$ times the reference uniform dipole of the same length and bending angle, has a fractional length $L_{0} / L$ given by the curves (red, blue, and green for TME, AME, and EME, respectively), and contributes $\left(L_{0} / L\right) \kappa$ percentage to the bending angle. The end field is weaker by a factor of $r$, which is plotted in Figs. 3, 8, and 12.

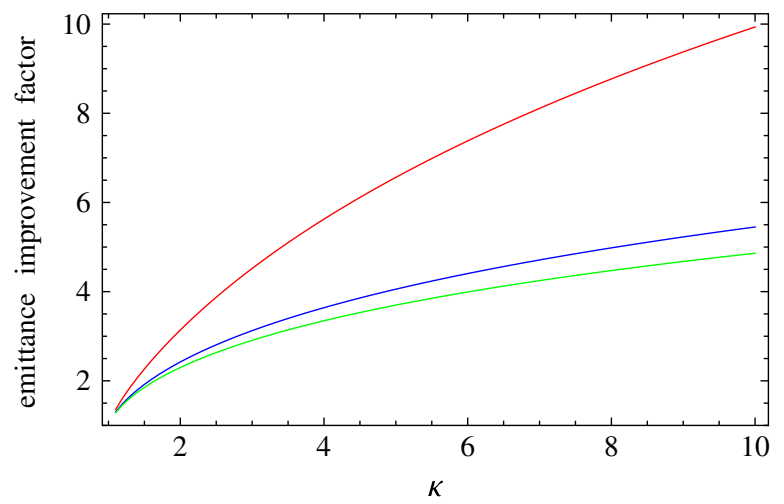

FIG. 18. Summary of improvement factors in the theoretical minimum emittances for TME (red), AME (blue), and EME (green) lattices, resulting from the above optimal profiles.

It is clear that, compared to the conventional uniform dipoles, a significant reduction in theoretical minimum emittance can be achieved with optimal bending profiles. However, there are challenges to implementing theoreticalminimum-emittance lattices mainly due to the stringent requirement of small beta function, even for uniform dipoles. Nonetheless, it is still possible to gain even with beta function mismatch. For example, if one starts with a minimum emittance lattice with uniform dipoles, then replaces them with optimal dipoles but keeps the same beta function, the gain in theoretical minimum emittance is over $\kappa$, but the loss in TME emittance due to beta function mismatch is given by $(\tilde{\beta}+1 / \tilde{\beta}) / 2 \sim \kappa / 2$. Thus, the net TME emittance reduction is about a factor of 2. Further reduction has to rely on a better matched beta function. Since the minimum beta is proportional to dipole length, one may consider increasing dipole length, but there are costs and other limitations to consider.

This paper explored the emittance reduction for a given bending angle. The cubic dependence of emittance on the bending angle motivated the development of multibend-achromat lattice for future light sources using unconventional magnets $[16,17]$. It will be interesting to see whether a combination of both approaches can yield lower emittance and/or better lattices. Strong natural chromaticity is a challenge for all low emittance lattice and thus deserves systematic analysis, which is beyond the scope of this paper.

\section{ACKNOWLEDGMENTS}

The author W. W. Ho would like to acknowledge support from the 2011 Lee Teng Scholarship Program at Argonne National Laboratory and Fermi National Accelerator Laboratory, during which most of the detailed computations were carried out [18]. This work was supported by U.S. Department of Energy, Office of Science, Office of Basic Energy Sciences, under Contract No. DE-AC0206CH11357.

\section{APPENDIX A: INTEGRALS USED IN THE COMPUTATION}

The integrals used to compute $\langle\langle\boldsymbol{\xi}\rangle\rangle$ and $\left\langle\left\langle\boldsymbol{\xi} \boldsymbol{\xi}^{T}\right\rangle\right\rangle$ are listed here:

$$
\begin{gathered}
\int_{0}^{L} \frac{d s}{\rho^{2}}=\frac{L_{0}}{\rho_{0}^{2}}\left[1+\frac{r-1}{g r}\right], \\
\int_{0}^{L} \frac{d s}{\rho^{3}}=\frac{L_{0}}{\rho_{0}^{3}}\left[1+\frac{r^{2}-1}{2 g r^{2}}\right], \\
\int_{0}^{L} \frac{\hat{\xi}_{p} d s}{\rho^{3}}=\frac{L_{0}^{2}}{2 \rho_{0}^{4}}\left[1+\frac{(1+2 g)\left(r^{2}-1\right)-2 \ln r}{2 g^{2} r^{2}}\right],
\end{gathered}
$$




$$
\begin{gathered}
\int_{0}^{L} \frac{\hat{\xi} \hat{\xi}_{p} d s}{\rho^{3}}=-\frac{L_{0}^{4}}{8 \rho_{0}^{5}}\left\{1+\frac{4(g+1)(r-1)^{2}+g^{2}(2 g+3)\left(r^{2}-1\right)-2\left[3 g^{2}+4 r-4+2(g-1) \ln r\right] \ln r}{g^{4} r^{2}}\right\}, \\
\int_{0}^{L} \frac{\hat{\xi}^{2} d s}{\rho^{3}}=\frac{L_{0}^{5}}{20 \rho_{0}^{5}}\left\{1-\frac{5\left\{x(1-r)+4\left[g^{3}+3+4 g(r-1)-2 r(r+2)\right] \ln r+4(g-1)^{2}(\ln r)^{2}\right\}}{2 g^{5} r^{2}}\right\} .
\end{gathered}
$$

where $x=10-22 r+g(2+g)\left[g^{2}-4+\left(g^{2}+4\right) r\right]$.

\section{APPENDIX B: EXPRESSIONS FOR THE $c$ PARAMETER}

The $c$ parameter for a dipole starting with a constant bending radius followed by a linear ramp to the end is given by Eq. (42), where the messy subexpressions are listed here:

$$
\begin{gathered}
c_{1}=36\left\{2 g^{8} r^{4}-g^{7} r^{2}\left(7 r^{2}-1\right)-15(r-1)^{2}\left[2 g\left(13 r^{2}-2 r+1\right)+7 r^{2}-2 r-1\right]\right\} \\
\left.c_{2}=36 g^{2}\left\{g^{3} r^{2}\left(99 r^{2}+18 r-1\right)-5 g(3 r-1)\left(41 r^{3}+25 r^{2}+3 r+3\right)-30\left(24 r^{4}-11 r^{2}-2 r+1\right)\right]\right\} \\
c_{3}=3 g^{4}\left[2 g^{2} r^{2}\left(151 r^{3}-49 r^{2}-21 r-21\right)-15\left(167 r^{5}-65 r^{4}-100 r^{3}-52 r^{2}-3 r-3\right)\right] \\
c_{4}=4320 r^{2}(\ln r)^{2}+12 r^{2} \ln r c_{5}+36 r c_{6} \\
c_{5}=540\left(r^{2}-1\right)+5 \\
c_{6}\left(216+216 g-12 g^{2}-39 g^{3}-24 g^{4}+16 g^{5}\right) r^{2}+3 g\left(-360+20 g^{2}+35 g^{3}-16 g^{4}\right) \\
+60\left(2-2 g+g^{2}\right)+\left(510+360 g+360 g^{2}-100 g^{4}+6 g^{5}+7 g^{6}\right) r \\
-\left(60+480 g+840 g^{2}+560 g^{3}-100 g^{4}-154 g^{5}+17 g^{6}+12 g^{7}\right) r^{3} \\
+20\left(1+2 g+2 g^{2}\right)\left(6+6 g+3 g^{2}-4 g^{3}\right) r^{4}+30\left(1+2 g+2 g^{2}\right)^{2} r^{5} .
\end{gathered}
$$

[1] M. Sommer, Internal Report No. DCI/NI/20/81, 1981; see references in [4] for more internal reports in the early years.

[2] L. Teng, FNAL Report No. FNAL/TM-1269, 1984; ANL Report No. LS-17, 1985.

[3] D. Trbojevic and E. Courant, in Proceedings of the Fourth European Particle Accelerator Conference EPAC 94, London, England (World Scientific, River Edge, NJ, 1994), p. 1000.

[4] S. Y. Lee, Phys. Rev. E 54, 1940 (1996).

[5] H. Tanaka and A. Ando, Nucl. Instrum. Methods Phys. Res., Sect. A 369, 312 (1996).

[6] Y. Shoji and A. Ando, Nucl. Instrum. Methods Phys. Res., Sect. A 425, 377 (1999).

[7] J. Guo and T. Raubenheimer, in Proceedings of the 8th European Particle Accelerator Conference, Paris, 2002 (EPS-IGA and CERN, Geneva, 2002), p. 1136.

[8] Y. Papaphilippou and P. Elleaume, in Proceedings of the 21st Particle Accelerator Conference, Knoxville, 2005 (IEEE, Piscataway, NJ, 2005), p. 2086.

[9] R. Nagaoka and A. F. Wrulich, Nucl. Instrum. Methods Phys. Res., Sect. A 575, 292 (2007).
[10] A. Streun, PSI Internal Report No. SLS-TME-TA-20060297, 2007.

[11] C.-x. Wang, Phys. Rev. ST Accel. Beams 12, 061001 (2009).

[12] C.-x. Wang, Y. Wang, and Y. Peng, Phys. Rev. ST Accel. Beams 14, 034001 (2011).

[13] Y. Jiao, Y. Cai, and A.W. Chao, Phys. Rev. ST Accel. Beams 14, 054002 (2011).

[14] P. Emma and T. Raubenhemier, Phys. Rev. ST Accel. Beams 4, 021001 (2001).

[15] C.-x. Wang, Phys. Rev. E 74, 046502 (2006).

[16] S. C. Leemann et al., Phys. Rev. ST Accel. Beams 12, 120701 (2009).

[17] S. C. Leemann and A. Streun, Phys. Rev. ST Accel. Beams 14, 030701 (2011).

[18] W. W. Ho and C.-x. Wang, http://www.illinoisacceleratorinstitute.org/2011 Program/student_papers/Wen_Wei_Ho .pdf. More general linear-ramped profiles are investigated in this report. Although analytical expressions are obtained in MATHEMATICA, they are too messy due to the number of free parameters involved to describe the geometry. 